\title{
Synthesis of Deceptive Strategies in Reachability Games with Action Misperception
}

\author{
Abhishek N. Kulkarni and Jie Fu \\ Worcester Polytechnic Institute, Worcester, USA \\ \{ankulkarni, jfu2\}@wpi.edu
}

\begin{abstract}
We consider a class of two-player turn-based zerosum games on graphs with reachability objectives, known as reachability games, where the objective of Player 1 (P1) is to reach a set of goal states, and that of Player $2(\mathrm{P} 2)$ is to prevent this. In particular, we consider the case where the players have asymmetric information about each other's action capabilities: P2 starts with an incomplete information (misperception) about P1's action set, and updates the misperception when P1 uses an action previously unknown to P2. When P1 is made aware of $\mathrm{P} 2$ 's misperception, the key question is whether $\mathrm{Pl}$ can control $P 2$ 's perception so as to deceive $P 2$ into selecting actions to Pl's advantage? To answer this question, we introduce a dynamic hypergame model to capture the reachability game with evolving misperception of $\mathrm{P} 2$. Then, we present a fixedpoint algorithm to compute the deceptive winning region and strategy for P1 under almost-sure winning condition. Finally, we show that the synthesized deceptive winning strategy is at least as powerful as the (non-deceptive) winning strategy in the game in which P1 does not account for P2's misperception. We illustrate our algorithm using a robot motion planning in an adversarial environment.
\end{abstract}

\section{Introduction}

Synthesis of winning strategies in reachability games is a central problem in reactive synthesis [Pnueli and Rosner, 1989], control of discrete event systems [Ramadge and Wonham, 1989], and robotics [Fainekos et al., 2009]. In a two-player reachability game, a controllable player, P1 (pronoun "she"), plays against an uncontrollable adversarial player, P2 (pronoun "he"), to reach the goal states. These games have been extensively studied in algorithmic game theory [de Alfaro et al., 2007] and reactive synthesis [Bloem et al., 2012]. Polynomial-time algorithms are known for synthesizing surewinning and almost-sure winning strategies, when both players have complete and symmetric information. However, the solution concepts for such games under asymmetric information have not been thoroughly studied.
Information asymmetry arises when a player has some private information that are not shared with others [Rasmusen, 1989]. We consider the case when $P 1$ has complete information about both players' action capabilities, but P2 starts with an incomplete information about P1's action capabilities. As two players interact, their information evolves. Particularly, when P1 uses an action previously unknown to P2, P2 can update his knowledge about the other's capabilities using an inference mechanism. In response, $\mathrm{P} 2$ would update his counter-strategy. We are interested in the following question: if $P 1$ is made aware of the initial information known to $P 2$ and his inference mechanism, can P1 find a strategy to control P2's information in such a way that P2's counterstrategy given his evolving information is advantageous to $P 1$ ? In the context of qualitative analysis of reachability games, this could be interpreted as: from a state that is losing for Pl in a game with symmetric information, can P1 reach his goal from the same state when the information is asymmetric? We note that a strategy of P1 that controls P2's information to P1's advantage is indeed deceptive [Ettinger and Jehiel, 2010]. In this paper, we establish that such a deceptive winning strategy may exist under almost-sure winning condition and propose an algorithm to synthesize it.

We approach the question by modeling the interaction between P1 and P2 as a hypergame [Bennett, 1977]. A hypergame allows players to play different games in their minds and further allows them to model the games that others might be playing. In literature, hypergames and Bayesian games [Harsanyi, 1967] are common models to capture game-theoretic interactions with asymmetric, incomplete information. In Bayesian games, each player uses his incomplete information to define a probability distribution over the possible types of the opponent. The distributions over types are assumed to be common knowledge. In hypergames, no such probabilistic characterizations of incomplete information is used or assumed. For action deception, $\mathrm{P} 2$ has incomplete information about P1's capabilities but does not have a prior knowledge about the set of possible types of P1. Thus, we adopt the hypergame model to understand action deception. In the past, hypergame model has been used to study deception [Gutierrez et al., 2015; Kovach, 2016]. These papers mainly focus on extending the notion of Nash equilibrium to level- $k$ normal form hypergames. [Gharesifard and Cortés, 2014] use the notion of 
H-digraph to establish necessary and sufficient conditions for deceivability. An H-digraph models a hypergame as a graph with nodes representing different outcomes in a normal-form game. However, our game model is not a normal-form game, but instead a game on graph. A hypergame model based on a game on graph has been defined in [Kulkarni and Fu, 2019] where one player has incomplete information about the opponent's task specification. However, their model assumes the perception of $\mathrm{P} 2$ to remain constant, whereas in our case, the information available to both players' may evolve as the game progresses. Given this background, we state the two key contributions of the paper.

Dynamic Hypergame Model for Action Misperception. We introduce a dynamic hypergame on graph model that captures (i) the evolving information of P2, and (ii) the P1's information regarding current perception of $\mathrm{P} 2$, in a single graphical model.

Deceptive Almost-sure Winning Synthesis Algorithm. Given a dynamic hypergame model, we propose an algorithm to compute deceptive almost-sure winning region, and thereby a deceptive almost-sure winning strategy for P1 that effectively exploits P2's misperception. We prove that the deceptive winning region is a superset of the (non-deceptive) winning region in the symmetric information game where P1 does not account for P2's misperception. This in turn implies that the deceptive winning strategy is at least as powerful as the (non-deceptive) winning strategy.

\section{Preliminaries}

Let $\Sigma$ be a finite alphabet. A sequence of symbols $w=$ $w_{0} w_{1} \ldots w_{n}$ with $w_{i} \in \Sigma, i=0,1, \ldots, n$ is called a finite word and $\Sigma^{*}$ is the set of finite words that can be generated with alphabet $\Sigma$. We denote by $\Sigma^{\omega}$, the set of $\omega$-regular words obtained by concatenating the elements in $\Sigma$ infinitely many times. Given a set $X$, let $\operatorname{Dist}(X)$ be the set of probability distributions over $X$. Given a distribution $\delta \in \operatorname{Dist}(X)$, the set $\operatorname{Supp}(\delta)=\{x \in X \mid \delta(x)>0\}$ is called the support of the distribution.

\subsection{Games on Graph}

Consider an interaction between two players; P1 with a reachability objective and P2 with an objective of preventing P1 from completing her task.

Definition 1 (Game on Graph). Let the action sets of $P 1$ and $P 2$ be $A_{1}$ and $A_{2}$, respectively. Then, a turn-based game on graph is the tuple

$$
\mathcal{G}=\langle S, A c t, T, F\rangle
$$

where

- $S=S_{1} \cup S_{2}$ is the set of states partitioned into P1's states, $S_{1}$, and P2's states, $S_{2}$. P1 chooses an action when $s \in S_{1}$ and $P 2$ chooses an action when $s \in S_{2}$.

- Act $=A_{1} \cup A_{2}$ is set of actions for P1 and P2.

- $T: S \times A c t \rightarrow S$ is a deterministic transition function that maps a state and an action to a successor state.

- $F \subseteq S$ is a set of final states.

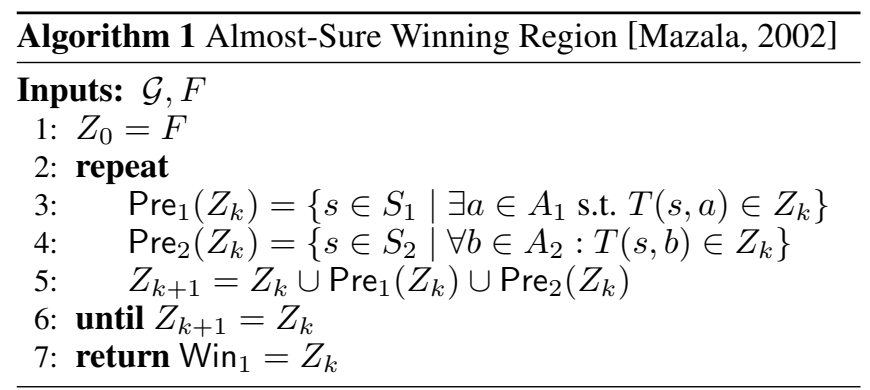

A trace in the game $\mathcal{G}$ is an infinite, ordered sequence of state-action pairs $\tau=\left(s_{0}, a_{0}\right),\left(s_{1}, a_{1}\right),\left(s_{2}, a_{2}\right), \ldots$ We write $\tau[n]=\left(s_{n}, a_{n}\right)$ to denote $n$-th state-action pair. A run $\rho$ is the projection of trace onto the state space. We denote it as the sequence $\rho=\tau L_{S}=s_{0} s_{1} s_{2} \ldots$. Similarly, the actionhistory is the projection of trace onto the action space, denoted by $\alpha=\tau l_{A c t}=a_{0} a_{1} a_{2} \ldots$ The $k$-th element in a run (resp. action-history) is denoted by $\rho_{k}$ (resp. $\alpha_{k}$ ).

In this paper, we consider reachability objectives for $\mathrm{P} 1$. The set of states that occur in a run is given by $\operatorname{Occ}(\rho)=$ $\left\{s \in S \mid \exists k \in \mathbb{N} \cdot s=\rho_{k}\right\}$. A run $\rho$ is said to be winning for $\mathrm{P} 1$ in the reachability objective if it satisfies $\operatorname{Occ}(\rho) \cap F \neq \emptyset$. If a run is not winning for $\mathrm{P} 1$, then it is winning for $\mathrm{P} 2$.

The memoryless stochastic or randomized strategies for P1 and $\mathrm{P} 2$ are defined as $\pi: S_{1} \rightarrow \operatorname{Dist}\left(A_{1}\right)$ and $\sigma: S_{2} \rightarrow$ $\operatorname{Dist}\left(A_{2}\right)$, respectively. As the model in Def. 1 is a deterministic turn-based game model, it is sufficient to consider memoryless strategies [Mazala, 2002]. Let $\Omega_{s}^{\pi, \sigma}$ be the exhaustive set of runs that result when $\mathrm{P} 1$ and $\mathrm{P} 2$ play strategies $\pi$ and $\sigma$ in a game starting at the state $s \in S$. The randomized strategies of P1 and P2 induce a Markov chain from $\mathcal{G}$-that is, a probability distribution over the set $\Omega_{s}^{\pi, \sigma}$.

Given a state $s \in S$, a randomized strategy $\pi$ is almostsure winning (ASW) for P1, if and only if for every possible randomized strategy $\sigma$ of $\mathrm{P} 2$, the probability is one for a run that satisfies $\operatorname{Occ}(\rho) \cap F \neq \emptyset$, given the distribution of runs induced by $(\pi, \sigma)$. A state is called an almost-sure winning $(A S W)$ state for $\mathrm{P} 1$, if there exists an ASW strategy for P1 from that state. The exhaustive set of almost-sure winning states for $\mathrm{P} 1$ is called her $A S W$ region. The almost-sure winning region can be computed using Alg. 1.

Let us introduce a running example that we shall use to explain the concepts in this paper.

Example 1. Consider the game graph in Fig. 1. The circle states, $\left\{s_{1}, s_{3}\right\}$, are Pl states and the square states, $\left\{s_{0}, s_{2}\right\}$, are $P 2$ states. The objective of $P 1$ is to reach to the final state $s_{0}$ from the initial state $s_{2}$. Pl's action set is $A_{1}=\left\{a_{1}, a_{2}\right\}$, and $P 2$ 's action set is $A_{2}=\left\{b_{1}, b_{2}\right\}$.

The ASW region for $P 1$ in the game is $\operatorname{Win}_{1}=\left\{s_{0}, s_{1}\right\}$. This can intuitively be understood as follows. Pl can win from state $s_{1}$ by choosing the action $a_{1}$. However, the states $s_{2}$ and $s_{3}$ are losing for $P 1$ because $P 2$ has a strategy to indefinitely restrict the game within the states $s_{2}, s_{3}$ by choosing the action $b_{2}$ at the state $s_{2}$. 


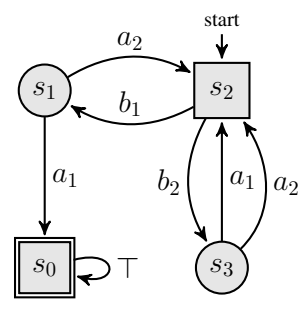

Figure 1: An example game on graph

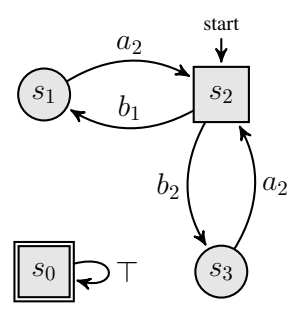

Figure 2: Perceptual game of P2

\subsection{Action Misperception and Information Asymmetry}

In this paper, we consider the sub-class of games with asymmetric information in which $\mathrm{P} 2$ has incomplete information about P1's action capabilities. This is formalized in the following assumption.

Assumption 1. Pl has complete information about the players' action sets, i.e. P1 knows $A_{1}$ and $A_{2}$. P2 only knows his own action set $A_{2}$, but (mis)perceives Pl's action set to be a subset $X \subsetneq A_{1}$. Both players have complete information about the game state space $S$ and the final states $F$.

The result of Assumption 1 is that $\mathrm{P} 1$ and $\mathrm{P} 2$, in their minds, play different games to synthesize their respective strategies. We refer to these games as the perceptual games of the players. P1's perceptual game is identical to the ground-truth game; $\mathcal{G}\left(A_{1}\right)=\left\langle S, A_{1} \cup A_{2}, T, F\right\rangle$, while P2's perceptual game is a game under misperception; $\mathcal{G}(X)=$ $\left\langle S, X \cup A_{2}, T, F\right\rangle$. Let us formalize the new notation used to distinguish between the perceptual games of $\mathrm{P} 1$ and $\mathrm{P} 2$.

Notation 1. Let $X \subseteq A_{1}$ be a subset of P1's action set. We denote a perceptual game in which P1's action set is $X$ by $\mathcal{G}(X)=\left\langle S, X \cup A_{2}, T, F\right\rangle$. The winning regions for $\mathrm{P} 1$ and $\mathrm{P} 2$ in the game $\mathcal{G}(X)$ are denoted by $\mathrm{Win}_{1}(X)$ and $\operatorname{Win}_{2}(X)$, respectively.

Assuming P1 and P2 to be rational players, they would use the solution approach reviewed in Section 2.1 to compute their winning strategies in their respective perceptual games. That is, $\mathrm{P} 1$ will solve $\mathcal{G}\left(A_{1}\right)$ in her mind to obtain $\pi$ and $\mathrm{P} 2$ will solve $\mathcal{G}(X)$ in his mind to compute $\sigma$. However, P1 is likely to compute a conservative strategy because she overestimates the information available to P2. Naturally, we want to know whether P1 can improve her strategy if she is made aware of $P 2$ 's current misperception $X$ ?

Before we answer the above question, recall from Section 1 that we allow P2's misperception to evolve during the game. For instance, what would happen when $\mathrm{P} 2$ observes P1 playing an action $a \in A_{1}$, which P2 did not believe to be in P1's action set? We might argue that P2 will at least add a new action $a$ to his perceived action set, $X$, of $\mathrm{P} 1$. Thus, the new perception would be $X \cup\{a\}$. Also, P2 might be capable of complex inference. That is, on observing that $\mathrm{P} 1$ can perform an action $a, \mathrm{P} 2$ might infer that P1 must be capable of actions $b$ and $c$, thus, updating his perception set to $X \cup\{a, b, c\}$. To capture such inference capabilities, we introduce a generic perception update function for $\mathrm{P} 2$ as follows,

Definition 2 (Inference Mechanism). A deterministic inference mechanism is a function $\eta: 2^{A_{1}} \times A_{1} \rightarrow 2^{A_{1}}$ that maps a subset of actions $X \subseteq A_{1}$ and an action $a \in A_{1}$ to an updated subset of actions $Y=\eta(X, a)$ such that $a \in Y$.

Given the formalism of inference mechanism to capture the evolving misperception of P2 during the game, we now state our problem statement.

\subsection{Problem Statement}

When P2's misperception evolves during the game, P1 should also strategize to reveal an action that is not currently known to P2. By doing so, P1 may control the evolution of P2's misperception to her advantage. Let us revisit Example 1 to develop an intuition of how P1 might control P2's perception.

Example 2 (Example 1 contd.). Suppose that, in Example 1, $P 2$ starts with a misperception about P1's action capabilities as $X_{0}=\left\{a_{2}\right\}$. In this setup, let us understand the perceptual games of the players. P1's perceptual game; $\mathcal{G}_{1}=\mathcal{G}\left(A_{1}\right)$, is the same as the ground-truth game as shown in Fig. 1. P2's perceptual game, initially, is the game $\mathcal{G}_{2}=\mathcal{G}\left(X_{0}\right)$ that does not include edges labeled with action $a_{1}$ as shown in Fig. 2. Clearly, as the final state $s_{0}$ is not reachable in $\mathcal{G}_{2}, P 2$ misperceives both actions $b_{1}$ and $b_{2}$ to be safe to play at state $s_{2}$, when only the action $b_{2}$ is safe in the ground-truth game.

When P1 is aware of P2's misperception, $X_{0}$, a deceptive strategy should, intuitively, not use $a_{1}$ unless the game state is $s_{1}$. Assuming P2 uses a randomized strategy with support $A_{2}$, it is easy to compute that the probability of reaching the state $s_{1}$ from the initial state $s_{2}$ is one. At $s_{1}$, Pl can win the game by choosing $a_{1}$ in one step. We note that if Pl uses $a_{1}$ in state $s_{3}$, then $P 2$ will update his perception to $X_{1}=A_{1}$, and mark the action $b_{1}$ to be unsafe in state $s_{2}$. Thus, Pl will never be able to win the game.

We call such a strategy of P1, where she intentionally controls P2's misperception, as an action-deceptive strategy or simply a deceptive strategy (see Def. 6 for a formal definition). We formalize our problem statement.

Problem 1. Consider a reachability game under information asymmetry in which Assumption 1 holds. If Pl is informed of the initial misperception of $P 2, X_{0}$, and his inference mechanism $\eta$, then determine a deceptive almost-sure winning strategy for P1 to satisfy her reachability objective.

In particular, we want to investigate whether the use of deception is advantageous for P1 or not. We say P1 gets advantage with deception if at least one game state that is not almost-sure winning for P1 in the game without deception becomes winning for her with use of deception. 


\section{Dynamic Hypergame for Action Deception}

When two players play different games in their minds, their interaction is better modeled as a hypergame [Bennett, 1977].

Definition 3 (First-level Hypergame). A first-level hypergame is defined as a tuple of the perceptual games being played by the players,

$$
\mathcal{H}^{1}=\left\langle\mathcal{G}_{1}, \mathcal{G}_{2}\right\rangle,
$$

where the P1 (resp. P2) solves the game $\mathcal{G}_{1}$ (resp. $\mathcal{G}_{2}$ ) to compute the winning strategy.

When one of the players is aware of the other player's perception, but the other player is not, we say that a second-level hypergame is being played. In line with Problem 1, we assume that $\mathrm{P} 1$ is aware of the $\mathrm{P} 2$ 's misperception, i.e. $\mathrm{P} 1$ knows the action set $X \subseteq A_{1}$ as perceived by $\mathrm{P} 2$. If $\mathrm{P} 1$ knows $X$, then $\mathrm{P} 1$ can construct the perceptual game of $\mathrm{P} 2, \mathcal{G}(X)$, and therefore $\mathrm{P} 1$ knows the first-level hypergame $\mathcal{H}^{1}$.

However, P2's perception evolves when he observes P1 using actions that are not included in $X$. This means that the game $\mathcal{G}(X)$ changes when P2's perception changes, and so does the hypergame $\mathcal{H}^{1}$. We now define a graph to model the hypergame representing the evolving misperception of $\mathrm{P} 2$, called as a dynamic hypergame on graph.

Definition 4 (Dynamic Hypergame on Graph). Let $\Gamma=2^{A_{1}}$. We define the dynamic hypergame on graph with action misperception as

where

$$
\mathcal{H}=\langle V, A c t, \Delta, \mathcal{F}\rangle,
$$

- $V=S \times \Gamma$ is the set of hypergame states,

- Act $=A_{1} \cup A_{2}$ is the set of actions of $P 1$ and $P 2$,

- $\Delta: V \times A c t \rightarrow V$ is the transition function such that $(s, X) \stackrel{a}{\rightarrow}\left(s^{\prime}, X^{\prime}\right)$ if and only if $s^{\prime}=T(s, a)$ and $X^{\prime}=$ $\eta(X, a)$,

- $\mathcal{F}=F \times \Gamma$ is the set of final states.

For convenience, we shall refer to the dynamic hypergame on graph as simply hypergame in the remainder of the paper. Analogous to game on graph, a trace in a hypergame is an infinite, ordered sequence of state-action pairs given by $\tau=\left(v_{0}, a_{0}\right)\left(v_{1}, a_{1}\right) \ldots$ and the action-history is defined as $\alpha=\tau L_{\text {Act }}=a_{0} a_{1} a_{2} \ldots$ In contrast with the game on graphs, we distinguish between a hypergame-run (h-run) as a projection of trace onto the hypergame state space $\nu=\tau L_{V}=v_{0} v_{1} v_{2} \ldots$ and a game-run as a projection of trace onto game state space $\rho=\tau \quad{ }_{S}=s_{0} s_{1} s_{2} \ldots$, where $s_{k}$ is the game state corresponding to hypergame state $v_{k}=\left(s_{k}, \cdot\right)$. A reachability objective is said to be satisfied over the hypergame if and only if $\operatorname{Occ}(\nu) \cap \mathcal{F} \neq \emptyset$, i.e. the hypergame-run $\nu$ visits a winning state in $\mathcal{F}$. By definition, the following statement is always true; $\operatorname{Occ}(\rho) \cap F \neq \emptyset$ if and only if $\operatorname{Occ}(\nu) \cap \mathcal{F} \neq \emptyset$.

Example 3 (Example 1 contd.). The hypergame modeling the asymmetric information from Example 2 is shown in Fig. 3 (the figure only shows the reachable states). Every state is represented as a tuple of a game state and the current misperception of $P 2$ at that

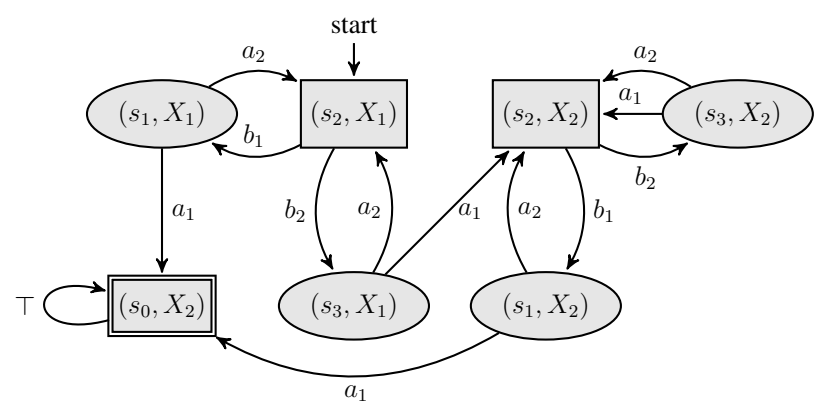

Figure 3: The dynamic hypergame on graph

state. Letting $X_{1}=\left\{a_{2}\right\}$ and $X_{2}=\left\{a_{1}, a_{2}\right\}$, the traces $\tau_{1}=\left(\left(s_{2}, X_{1}\right), b_{1}\right),\left(\left(s_{1}, X_{1}\right), a_{1}\right),\left(s_{0}, X_{2}\right)$, and $\tau_{2}=\left(\left(s_{2}, X_{1}\right), b_{2}\right),\left(\left(s_{3}, X_{1}\right), a_{1}\right),\left(\left(s_{2}, X_{2}\right), b_{1}\right)$, $\left(\left(s_{1}, X_{2}\right), a_{1}\right),\left(s_{0}, X_{2}\right)$ are the examples of winning traces for Pl in the hypergame. However, in the next section, we will see that $(a) \tau_{2}$ will never be observed when both players act rationally, whereas $\tau_{1}$ might be observed, and (b) how to identify all rationally possible winning traces for $P 1$.

\section{Synthesis of Deceptive Almost-Sure Winning Strategy}

In this section, we present an algorithm to synthesize deceptive almost-sure winning (DASW) strategies that are rationally possible in the hypergame. To do so, we must reason about $\mathrm{P} 2$ 's perception and his subjectively rational actions. To understand this, we revisit the concept of permissive strategies in a game on graph.

Recall that an action is permissive for a player at a given state if the player can stay within the winning region by performing that action [Bernet et al., 2002]. In a game under information asymmetry, whether a state is winning or not depends on the player's perception. Hence, we define the notion of perceptually permissive actions, which extends the definition of permissive actions to model evolving perception.

Definition 5 (Perceptually Permissive Actions of P2). Let $u=(s, X) \in V_{2}$ and $v=\left(s^{\prime}, X\right)$ be two hypergame states such that $v=\Delta(u, b)$ for some $b \in A_{2}$. Then, the set $M(u)=\left\{a \in A_{2} \mid s^{\prime} \in \mathrm{Win}_{2}(X)\right\}$ is the set of P2's perceptually permissive actions at $u$.

In words, the perceptually permissive actions for a given state $u=(s, X)$ is the set of permissive actions for $\mathrm{P} 2$ in the perceptual game with action set $X$.

Remark 1. By Def. 2, P2's action does not have any effect on $\mathrm{P} 2$ 's perception. Hence, the P2's perception at $v$ is the same as her perception at $u$, i.e. $X$.

Assumption 2. At a state $v \in V_{2}, P 2$ plays a randomized strategy, $\sigma$, defined over the perceptually permissive actions $M(v)$ such that $\operatorname{Supp}(\sigma(v))=M(v)$.

Now, we formalize the notion of Deceptive Almost-Sure Winning (DASW) strategy.

Definition 6 (Deceptive Almost-Sure Winning (DASW) Strategy). Given a hypergame state $v \in V$, a strategy $\pi$ is said to be deceptive almost-sure winning (DASW) strategy for 
$P 1$ if and only if for every P2's strategy $\sigma$ satisfying Assumption 2 , the probability of an $h$-run $\nu$ induced from $\mathcal{H}$ by $(\pi, \sigma)$ satisfying $\operatorname{Occ}(\nu) \cap \mathcal{F} \neq \emptyset$ is one.

The states at which P1 has a DASW strategy are called as DASW states. The exhaustive set of all DASW states is called DASW region.

Now, we discuss Alg. 2 that computes the DASW region for P1. Our algorithm is inspired by the algorithm presented in [de Alfaro et al., 2007] to compute the almost-sure winning (ASW) region in the concurrent $\omega$-regular games. The idea behind Alg. 2 is to identify the states where P2 perceives some unsafe actions as safe due to misperception. This is achieved by modifying the definitions of SAFE- 1 and SAFE2 in [de Alfaro et al., 2007] using the following definitions:

$$
\begin{aligned}
& \operatorname{DAPre}_{1}^{1}(U)=\left\{v \in V_{1} \mid \exists a \in A \text { s.t. } \Delta(v, a) \in U\right\}, \\
& \operatorname{DAPre}_{1}^{2}(U)=\left\{v \in V_{2} \mid \forall b \in M(v) \text { s.t. } \Delta(v, b) \in U\right\}, \\
& \operatorname{DAPre}_{2}^{1}(U)=\left\{v \in V_{1} \mid \forall a \in A \text { s.t. } \Delta(v, a) \in U\right\}, \\
& \text { DAPre }_{2}^{2}(U)=\left\{v \in V_{2} \mid \forall b \in M(v) \text { s.t. } \Delta(v, b) \in U\right\} .
\end{aligned}
$$

The Alg. 2 works as follows. It starts with $Z_{0}=$ $\operatorname{Win}_{1}\left(A_{1}\right) \times \Gamma$ from where P1 has an ASW strategy to win the game no matter of P2's perception. Then it iteratively expands the set by invoking SAFE- 2 followed by SAFE- 1 until a fixed-point is reached. The SAFE-1 sub-routine computes the largest subset $Y$ of the input set $U$, such that $\mathrm{P} 1$ has a strategy to restrict the game indefinitely within $Y$. SAFE-2 sub-routine computes the largest subset $Y$ of the input set $U$, such that given his current (mis)perception, $\mathrm{P} 2$ can restrict the game indefinitely within $Y$. Here, it is important to note that P2 chooses his actions based on his perceptual game $\mathcal{G}(X)$, and not the hypergame. Only P1 knows the hypergame because she is aware of P2's misperception. As a consequence, before reaching the fixed-point, SAFE-2 might include states from which $\mathrm{P} 2$ may not have a strategy to indefinitely restrict $\mathrm{P} 1$ from reaching $Z_{0}$, i.e. $\mathrm{P} 1$ may have a DASW strategy from these states. However, after reaching the fixed-point, say in the iteration $k$, we observe that all DASW states are included in $Z_{k}$. A DASW strategy can then be computed based on the proof of Thm. 1. Let us now revisit the Example 3 to understand Alg. 2.

Example 4 (Example 3 contd.). Consider the hypergame graph as shown in Fig. 3. Recall from Example 1 that Almost-Sure Winning (ASW) region is $\operatorname{Win}_{1}\left(A_{1}\right)=\left\{s_{0}, s_{1}\right\}$, therefore, we have $Z_{0}=\left\{\left(s_{0}, X_{2}\right),\left(s_{1}, X_{2}\right),\left(s_{1}, X_{1}\right)\right\}$ (we omit $\left(s_{0}, X_{1}\right)$ as it is unreachable). The perceptually permissive actions for $P 2$ are $M\left(\left(s_{2}, X_{1}\right)\right)=\left\{b_{1}, b_{2}\right\}$ and $M\left(\left(s_{2}, X_{2}\right)\right)=\left\{b_{2}\right\}$.

Iteration 1 of DASW. The first step is to compute $C_{0}$, i.e. the subset of $V \backslash Z_{0}$ which $P 2$ perceives to be safe for himself. The SAFE-2 sub-routine takes 3 iterations to reach a fixed-point, at the end of which $C_{0}=\left\{\left(s_{2}, X_{2}\right),\left(s_{3}, X_{2}\right)\right\}$. The next step is to compute $Z_{1}$, which the largest subset of $V \backslash C_{0}$ in which Pl can stay indefinitely. The SAFE-1 subroutine takes 2 iterations to reach a fixed point. In its first iteration, DAPre ${ }_{1}^{1}$ adds a state $\left(s_{3}, X_{1}\right)$ and DAPre ${ }_{1}^{2}$ adds a state $\left(s_{2}, X_{1}\right)$ to $Z_{1}$. The interesting observation here is that

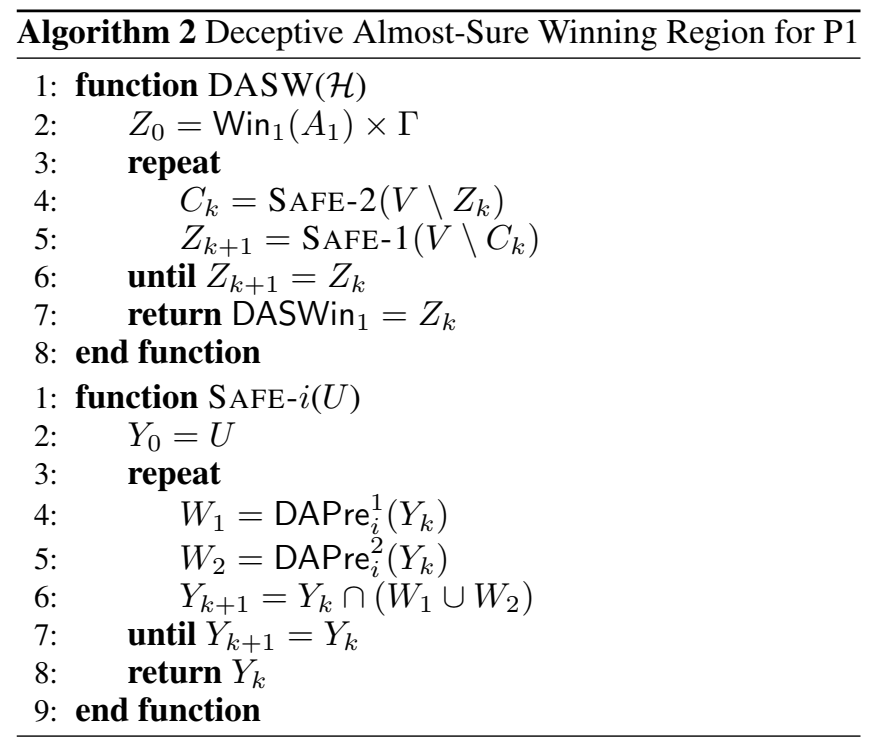

$\left(s_{2}, X_{1}\right)$ is added because the actions $b_{1}$ and $b_{2}$ are perceptually permissive actions for $P 2$, both of which lead to a state in $V \backslash C_{0}$.

Iteration 2 of DASW. The fixed-point of DASW algorithm is reached in this iteration with $Z_{2}=\left\{\left(s_{0}, X_{2}\right)\right.$, $\left.\left(s_{1}, X_{2}\right),\left(s_{1}, X_{1}\right),\left(s_{2}, X_{1}\right),\left(s_{3}, X_{1}\right)\right\}$. The states $\left(s_{2}, X_{1}\right)$ and $\left(s_{3}, X_{1}\right)$ are idenitifed as the DASW states for P1.

Using intuition from Example 4 with the observation that $\operatorname{Win}_{1}\left(A_{1}\right) \subseteq \mathrm{DASWin}_{1} L_{S}$ holds for every hypergame $\mathcal{H}$ by definition, we formalize our first key result. It establishes that using action deception could be advantageous to P1.

Proposition 1. There exists a hypergame $\mathcal{H}$ for which $\operatorname{Win}_{1}\left(A_{1}\right) \subsetneq \mathrm{DASWin}_{1} \mathrm{l}_{S}$.

Next, we proceed to prove the correctness of Alg. 2 by showing that from every state in DASWin ${ }_{1}$, we can construct a DASW strategy for P1 to ensure a visit to final states with probability one. We first prove two lemmas.

Lemma 1. In the $i$-th iteration of Alg. 2, P1 has a strategy to restrict the game indefinitely within $Z_{i}$, for all states in $Z_{i}$.

Proof. $\left(v \in V_{2}\right)$. For a P2's state in $Z_{i}$, every state $v^{\prime}=$ $\Delta(v, b)$ for a perceptually permissive action $b \in \mu(v)$ of P2 is in $Z_{i}$, by definition of DAPre ${ }_{1}^{2}$. Hence, no action of P2 at any state $v \in Z_{i}$ can lead the game state outside $Z_{i}$.

$\left(v \in V_{1}\right)$. For every P1's state in $Z_{i}$, there exists an action $a \in A$ such that the successor $v^{\prime}=\Delta(v, a)$ is in $Z_{i}$, by definition of DAPre ${ }_{1}^{1}$. Hence, P1 always has an action, consequently a strategy, to stay within $Z_{i}$.

Lemma 2. For every state $v \in Z_{i+1} \backslash Z_{i}$ added in the $i$-th iteration of Alg. 2, there exists an action that leads into $Z_{i}$.

Proof. Given any state $v \in V$ at the beginning of the $i$-th iteration, observe that it would belong to either $C_{i-1}, Z_{i}$ or $V \backslash\left(C_{i-1} \cup Z_{i}\right)$. We will prove the statement by showing that the every new state added to $Z_{i+1}$ has at least one transition into $Z_{i}$.

Consider $i$-th iteration of Alg. 2. The sub-routine SAFE-2 will add a P1 state $v \in V_{1} \backslash Z_{i}$ to $C_{i}$ if all the actions of P1 
stay within $V \backslash Z_{i}$. Similarly, SAFE-2 will include a P2 state $v \in V_{2} \backslash Z_{i}$ in $C_{i}$ if all perceptually permissive actions of $\mathrm{P} 2$ lead to a state within $V \backslash Z_{i}$. Therefore, a state that is not included in $C_{i}$ must have at least one action leading outside $V \backslash Z_{i}$, i.e. entering $Z_{i}$. In the next step, the sub-routine SAFE- 1 may add new states to $Z_{i+1}$ from the set $V \backslash C_{i}$. But, all states in $V \backslash C_{i}$ have an action entering $Z_{i}$. Hence, all new states added to $Z_{i+1}$ satisfy the statement.

Lma. 2 shows that $\mathrm{P} 1$ has a strategy to reach $Z_{i}$ from a state added to $Z_{i+1}$ in one-step. However, this is not true for P2. From a P2 state in $Z_{i+1}$, there exists a positive probability to reach $Z_{i}$ because of Assumption 2. In the next theorem, we prove a stronger statement that from every state in $Z_{i+1}, \mathrm{P} 1$ can reach not only $Z_{i}$ but also $Z_{0}$ with probability one.

Theorem 1. From every state $v \in \mathrm{DASWin}_{1}, P 1$ has a $D A S W$ strategy to satisfy $\varphi$.

Proof. For any $v \in Z_{i}, i>1, \mathrm{P} 1$ has a strategy to stay within $Z_{i}$ indefinitely, by Lma. 1 . Furthermore, by Lma. 2 , the probability of reaching to a state $v^{\prime} \in Z_{i-1}$ from $v$ is strictly positive. Thus, given a run of infinite length, the probability of reaching $Z_{i-1}$ from $Z_{i}$ is one. By repeatedly applying this argument, the probability of reaching $Z_{0}$ from $Z_{i}$ is one.

The DASW strategy can be constructed based on the proof of Thm. 1. At a P1 state $v \in V_{1}$, if $i \geq 1$ is the smallest integer such that $v \in Z_{i}$, then $\pi(v)=\left\{a \in A_{1} \mid v^{\prime}=\right.$ $\Delta(v, a)$ and $\left.v^{\prime} \in Z_{i-1}\right\}$ is the DASW strategy of P1 at $v$. Given $\pi(v)$ is a set, P1 can select any action from this set. We also state the following two important corollaries (proofs omitted due to space) that follow from Prop. 1 and Lma. 2.

Corollary 1.1. For every $i \geq 0$, we have $Z_{i} \subseteq Z_{i+1}$.

Corollary 1.2. The projection of DASW region onto the game states is a superset of the ASW region.

Following the approach used in [de Alfaro et al., 2007], we note the complexity of Alg. 2 is quadratic in the size of $V$.

\section{An Illustrative Example}

We present a robot motion planning example over a $4 \times 4$ gridworld, shown in Fig. 4, to illustrate how a robot (P1) may use action deception in presence of an adversary (P2). The objective of the robot is to visit the two cells $(3,1)$ and $(3,3)$ containing the flags, while the task of the adversary is to prevent this. The readers familiar with Linear Temporal Logic (LTL) may recognize the above objective as a co-safe LTL specification $\diamond G_{1} \wedge \diamond G_{2}$. The action set of the robot is $A_{1}=\{\mathrm{N}, \mathrm{E}, \mathrm{S}, \mathrm{W}, \mathrm{NE}, \mathrm{NW}, \mathrm{SW}\}$ while that of adversary is $A_{2}=\{\mathrm{N}, \mathrm{E}, \mathrm{S}, \mathrm{W}\}$, where $\mathrm{N}, \mathrm{E}, \mathrm{S}, \mathrm{W}$ stand for north, east, south and west. At the start of the game, the adversary has incomplete information about the robot's action set as $X_{0}=\{\mathrm{N}, \mathrm{E}, \mathrm{S}, \mathrm{W}\}$. When the adversary observes the robot performing any of the actions from $\{\mathrm{NE}, \mathrm{NW}, \mathrm{SW}\}$, he updates his perception to $X_{1}=A_{1}$.

A game on graph representing above scenario can be constructed using the product operation given in [Baier and Katoen, 2008, Def. 4.16]. Every game state is a tuple $\left(x_{1}, y_{1}, x_{2}, y_{2}, t, q\right)$ where $x_{i}, y_{i}$ for $i=1,2$ denote the cell that $\mathrm{P} 1$ and $\mathrm{P} 2$ occupy, $t$ represents the player who chooses the next move and $q$ denotes a state of a deterministic finite

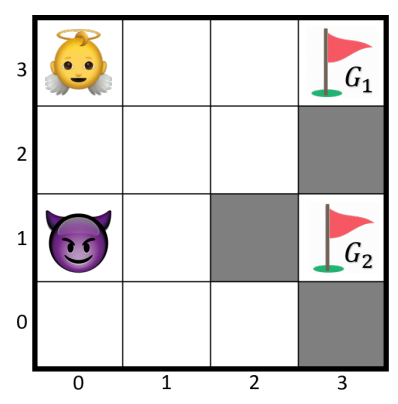

Figure 4: A game between P1 (angel) and P2 (demon).

automaton that keeps track of the progress $\mathrm{P} 1$ has made towards completion of her objective. The resulting game has $4^{4} \times 2 \times 4=2048$ states. We mark the states where P1 or P2 collide with an obstacle or with each other as the losing states for both players and, therefore, any action that leads to such states is disabled. Given the game on graph, a hypergame graph is constructed according to Def. 4 . The hypergame graph has $2048 \times 2=4096$ states because the adversary has two information states; $X_{0}$ and $X_{1}$.

When Alg. 2 is applied to the above hypergame graph, 2106 out of 4096 states are identified as DASW states. The projection of DASW states onto game state space results in 1172 states, while the ASW region has the size of 934 states. This means that $1172-934=238$ game states that were not almost-sure winning for P1 became winning for her, when P1 uses the DASW strategy.

\section{Discussion and Conclusion}

In this paper, we introduce a hypergame model to represent the interactions between two players with asymmetric information about their action capabilities. Given this model, we present an algorithm to synthesize action-deceptive strategies in a two-player turn-based zero-sum reachability game, where P2 starts with incomplete information about the P1's action capabilities. The synthesized strategy has two desirable properties. First, the DASW strategy is guaranteed to satisfy the reachability objective with probability one. Second, it is at least as powerful as the ASW strategy, because the DASW region is a superset of the ASW region. It worth noting that another game model of asymmetric information is Bayesian game, in which players have a Bayesian view of the types of others given their incomplete information. Our choice of hypergame model over Bayesian game model is motivated by the fact that a hypergame does not require consistency of priors that is assumed by Bayesian games [Halpern, 2002; Morris, 1995], and it also allows us to consider non-Bayesian players. Future work will focus on investigating action deception within hypergames with temporal logic payoffs and partial observations-that is, players have imperfect information about the history of games.

\section{Acknowledgements}

This material is based upon work supported by the Defense Advanced Research Projects Agency (DARPA) under Agreement No. HR00111990015. 


\section{References}

[Baier and Katoen, 2008] Christel Baier and Joost-Pieter Katoen. Principles of model checking. MIT press, 2008.

[Bennett, 1977] PG Bennett. Toward a theory of hypergames. Omega, 5(6):749-751, jan 1977.

[Bernet et al., 2002] Julien Bernet, David Janin, and Igor Walukiewicz. Permissive strategies: from parity games to safety games. RAIRO - Theoretical Informatics and Applications, 36(3):261-275, 2002.

[Bloem et al., 2012] Roderick Bloem, Barbara Jobstmann, Nir Piterman, Amir Pnueli, and Yaniv Sa'ar. Synthesis of reactive(1) designs. Journal of Computer and System Sciences, 78(3):911 - 938, 2012. In Commemoration of Amir Pnueli.

[de Alfaro et al., 2007] Luca de Alfaro, Thomas A Henzinger, and Orna Kupferman. Concurrent reachability games. Theoretical Computer Science, 386(3):188-217, 2007.

[Ettinger and Jehiel, 2010] David Ettinger and Philippe Jehiel. A theory of deception. American Economic Journal: Microeconomics, 2(1):1-20, February 2010.

[Fainekos et al., 2009] Georgios E. Fainekos, Antoine Girard, Hadas Kress-Gazit, and George J. Pappas. Temporal logic motion planning for dynamic robots. Automatica, 45(2):343 - 352, 2009.

[Gharesifard and Cortés, 2014] Bahman Gharesifard and Jorge Cortés. Stealthy deception in hypergames under informational asymmetry. IEEE Trans. Syst. Man, Cybern. Syst., 44(6):785-795, 2014.

[Gutierrez et al., 2015] Christopher N Gutierrez, Saurabh Bagchi, H Mohammed, and Jeff Avery. Modeling Deception In Information Security As A Hypergame-A Primer. In Proc. 16th Annu. Inf. Secur. Symp., page 41. CERIASPurdue University, 2015.

[Halpern, 2002] Joseph Y. Halpern. Characterizing the common prior assumption. Journal of Economic Theory, 106(2):316 - 355, 2002.

[Harsanyi, 1967] John C. Harsanyi. Games with Incomplete Information Played by "Bayesian" Players, I-III Part I. The Basic Model. Management Science, 1967.

[Kovach, 2016] Nicholas S Kovach. A Temporal Framework for Hypergame Analysis of Cyber Physical Systems in Contested Environments. Technical report, Air Force Institute of Technology, 2016.

[Kulkarni and Fu, 2019] Abhishek Ninad Kulkarni and Jie Fu. Opportunistic synthesis in reactive games under information asymmetry. CoRR, abs/1906.05847, 2019.

[Mazala, 2002] René Mazala. Infinite Games, pages 23-38. Springer Berlin Heidelberg, Berlin, Heidelberg, 2002.

[Morris, 1995] Stephen Morris. The common prior assumption in economic theory. Economics and Philosophy, 11(2):227-253, 1995.

[Pnueli and Rosner, 1989] A Pnueli and R Rosner. On the synthesis of a reactive module. pages 179-190, 1989.
[Ramadge and Wonham, 1989] P. J. G. Ramadge and W. M. Wonham. The control of discrete event systems. Proceedings of the IEEE, 77(1):81-98, Jan 1989.

[Rasmusen, 1989] Eric Rasmusen. Games and information: An introduction to game theory. Number 519.3/R22g. Blackwell Oxford, 1989. 